\section{So You're Trying to Choose a Confocal Microscope}

Ted Inoue, Video Microscopy, Inc.

If you are one of the many people who are currently considering the purchase of a confocal microscope, you may be asking yourself, "How can I be sure that the microscope I choose is the right one?"

Before proceeding, take a step back. Ask yourself: "Why use a confocal?" A confocal microscope is, by definition, a microscope which optically "slices" the sample by showing only those details which are at or near the plane of focus. This results in a clearer image than might be possible with a conventional (also called "wide-field") fluorescence microscope - a microscope in which every pixel in every image is corrupted by light from its neighbors. The optical slicing of the sample done by the confocal microscope lets you construct a 3-D representation of the sample that can be very useful for image visualization or analysis.

However, some confocal systems provide features that are unrelated to their confocality. In reviewing your needs, it may in fact be some of these features which are critical for your application. Therefore, determining the primary use for the confocal microscope should be your first consideration.

Ten years ago, answering "which confocal" was easy, as there was only one commercially available confocal microscope suitable for biological studies. Today, there are many vendors, each with a variety of products. To complicate matters further, there are now a variety of technologies that give the optical sectioning capability for which one typically uses a confocal microscope. The intent of this article is to help you determine the most appropriate technology for your primary application.

\section{What are the confocal technologies?}

Point scanner - The most common type of confocal microscope uses a scanned point from a laser. It achieves confocality by scanning an image of a small diaphragm or pinhole onto the specimen. This projection is then re-imaged by the microscope and passed through another diaphragm (called the exit pinhole) and detected by a light detector such as a photomultiplier tube or photodiode.

Resonant galvo point scanner - This variant of the point scanner achieves higher speeds by the use of a resonating galvanometer for the fast horizontal line scan rather than a conventional galvanometer which is limited to roughly $1000 \mathrm{~Hz}$. Because the resonance is tuned to a specific frequency, such scanners tend to be less versatile than normal point scanners.

Two photon - The mechanics of a two photon confocal are the same as the point scanner, except that they use a very intense beam of light at roughly double the excitation wavelength of the fluorechrome. At the exact plane of focus, the photon flux can be high enough for two photons to excite a fluorescent molecule simultaneously, causing it to go into its excited state and subsequently emit a photon. No exit diaphragm is necessary because any light emitted from the sample is known to be emanating from the excited point.

Line scanner - Considered a "partial confocal" because the microscope scans the sample with a line rather than a point. The line scanner achieves confocality along a single axis. What you get in return is (usually) a simpler design and video rate image acquisition.

Nipkow disk - Conceptually, the spinning disk confocal is just like a point scanner. The difference is that a disk containing a multitude of pinholes scans many points in the sample simultaneously. This allows the Nipkow type confocal to collect images significantly faster than a conventional point scanner. An added benefit of the Nipkow disk system is that it allows one to view the "live" confocal image.

Image Deconvolution - While deconvolution is not a confocal technique, it can yield very similar results and so warrants some discussion. Very roughly, deconvolution attempts to remove optical aberrations and interference from out-of-focus structures. Because it normally processes conventional wide-field fluorescence images, deconvolution can be a very cost-effective solution for obtaining high quality 3-D image sets.

\section{Which technology is best?}

As with most choices, choosing a single instrument for multiple applications is a compromise. Do you trade speed for spatial resolution and noise? Do you sacrifice flexibility for ease of use? Do you tolerate average results in many applications or demand excellent results in one? Only you can answer these questions.

To help you answer these questions, some of the key considerations are listed below.

\begin{tabular}{|c|c|c|c|c|c|c|}
\hline $\begin{array}{r}\text { Feature } \\
\text { Technology }\end{array}$ & $\begin{array}{c}\text { Acquisition speed } \\
\text { (Samples/sec) }\end{array}$ & $\begin{array}{c}\text { Resolution } \\
\qquad(x-y)\end{array}$ & Photo-damage & $\begin{array}{c}\text { Excitation Wave- } \\
\text { lengths }\end{array}$ & Cost & Ease of use \\
\hline Point scanning & $\begin{array}{l}\text { Full image: }-1 \\
\text { One line: } \sim 1000\end{array}$ & $\begin{array}{l}\text { Up to } 1.5 x \text { the resolution } \\
\text { of a wide-field micro- } \\
\text { scope }\end{array}$ & $\begin{array}{l}\text { Depends on scanning speed } \\
\text { and illumination levels }\end{array}$ & $\begin{array}{l}\text { Limited by choice of } \\
\text { laser }\end{array}$ & $\$ \$ \$$ & $\begin{array}{l}\text { Typically poor. Computer use and many adjustments } \\
\text { hecessary in order to acquire a single image }\end{array}$ \\
\hline Resonant galvo & $\begin{array}{l}\text { Full image: } \sim 30 \\
\text { One line: } \sim 15000\end{array}$ & $"$ & $\begin{array}{l}\text { Typically low because of high } \\
\text { scanning speed }\end{array}$ & $=$ & $\$ \$ \$ \$$ & $\begin{array}{l}\text { Slightly better than point scanning because high } \\
\text { speed allows rapid scanning and focusing }\end{array}$ \\
\hline $\begin{array}{l}\text { Two photon } \\
\text { point scanning }\end{array}$ & $\begin{array}{l}\text { Full image: }-1 \\
\text { One line: }-1000\end{array}$ & $\begin{array}{l}\text { Roughly the same as a } \\
\text { wide-field microscope }\end{array}$ & $\begin{array}{l}\text { Can be very low due to the } \\
\text { use of long wavelength excita- } \\
\text { ion but can be high if sample } \\
\text { absorbs excitation wavelength }\end{array}$ & " & $\$ \$ \$$ & $\begin{array}{l}\text { Slightly better than point scanning because no exit } \\
\text { pinhole alignment required, Significantly worse than } \\
\text { point scanning because complex laser system } \\
\text { heeded for illumination. } \\
\text { This system is currently only viable for highly } \\
\text { technical users }\end{array}$ \\
\hline Line scanning & $\begin{array}{l}\text { Full image: }-30 \\
\text { One line: }>15000 \\
\end{array}$ & $\begin{array}{l}\text { Slightly better than a } \\
\text { wide-field microscope }\end{array}$ & & . & $\$ \$ \$$ & $\begin{array}{l}\text { Somewhat better than point scanning because of few } \\
\text { adjustments and rapid scanning and focusing }\end{array}$ \\
\hline Nipkow disk & $\begin{array}{l}\text { Full image: depends on } \\
\text { disk speed. Ranges from } \\
\text { 30 to } 1000 \text {. }\end{array}$ & $\begin{array}{l}\text { Roughly the same as a } \\
\text { wide-field microscope }\end{array}$ & & " & $\$ \$$ & $\begin{array}{l}\text { Much better than all other technologies because of } \\
\text { minimal adjustments, no computer necessary, and } \\
\text { feal time viewing. }\end{array}$ \\
\hline Deconvolution & $\begin{array}{l}\text { Depends on image sen- } \\
\text { sor. }\end{array}$ & $\begin{array}{l}\text { Up to } 2 x \text { the resolution of } \\
\text { a wide-field microscope }\end{array}$ & $\begin{array}{l}\text { Determined by image acquisi- } \\
\text { tion system }\end{array}$ & $\begin{array}{l}\text { Determined by acquisi- } \\
\text { tion system }\end{array}$ & $\$$ & $\begin{array}{l}\text { Depends on acquisition system. Software can be } \\
\text { somewhat complex, requiring extensive user knowl- } \\
\text { edge and careful image collection }\end{array}$ \\
\hline
\end{tabular}




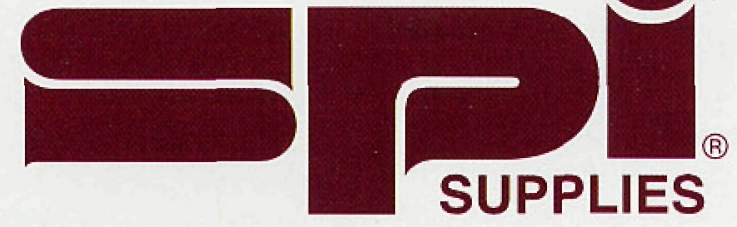

ह File Edit Uiew Go Bookmarks 0ptions Directory Help

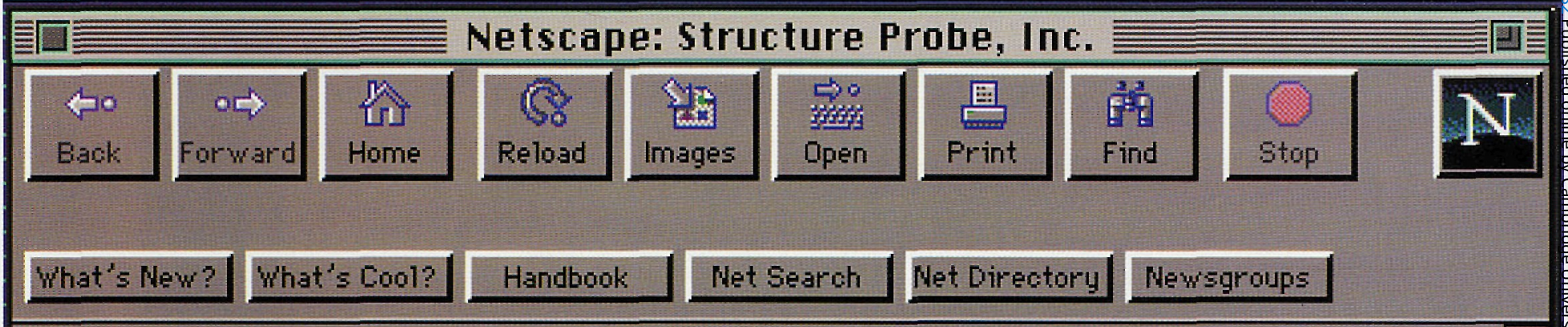

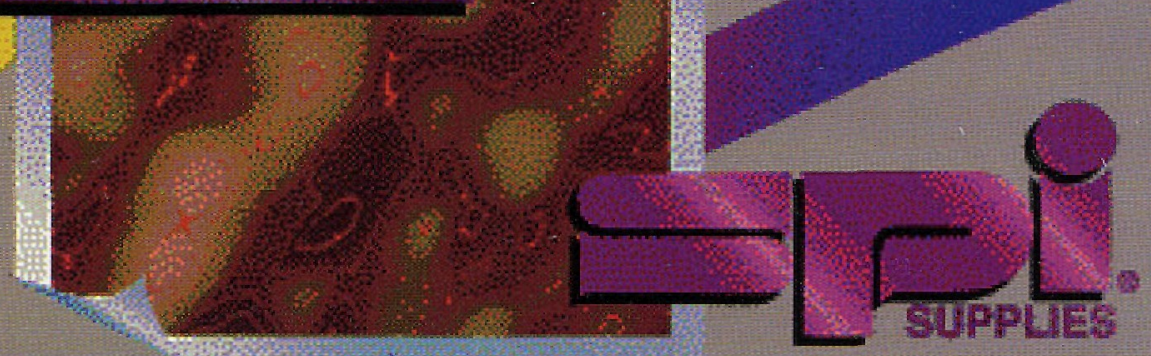

\section{Discover The SourceBook Online}

\section{http://www.2spi.com \\ Up-To-The-Minute Information \\ For All Your Microscopy Needs}

SPI Supplies Division of STRUCTURE PROBE, Inc.

P.O. Box 656 - West Chester, PA 19381-0656 USA 
So You're Trying to Choose a Confocal Microscope? Continued from page 20

\section{Image Acquisition Speed}

One of the less known, but extremely powerful features of the point scanning confocal microscope is the ability to capture some types of data quickly - at rates of 500 to thousands of samples per second. By repeatedly scanning a single line, the confocal captures events hundreds of times faster than it could by scanning the entire image.

This capability is extremely useful for measuring highspeed events such as muscle contraction. However, there is a clear tradeoff of spatial information for high speed. You can even imagine a situation in which the microscope samples a single point in the image at the full data rate of the microscope. For a point scanner, this can be from 500,000 to millions of samples per second. Most point scanning confocals can acquire high-speed data in this manner.

But what if you wish to maintain both spatial and temporal resolution? Fortunately, a Nipkow disk confocal using microlenses on each pinhole is efficient enough to acquire images with a temporal resolution on the order of milliseconds! How? By simply spinning the disk faster, the pinholes scan the entire image in a shorter time. Sounds great but there is a catch. The faster you move the pinholes, the less time you have to capture photons, resulting in a very dim image. Moreover, you need to supply a camera that is fast and sensitive enough to capture images at this rate.

\section{Image Resolution}

Are you looking at very fine filaments in a large structure?
Do you want to capture the finest possible image details?

While each technology is capable of acquiring images of very high resolution, in practice, single photon point scanners and deconvolution systems are highest resolution technologies.

Simple, single photon point scanning microscopes are capable of acquiring high-resolution images by virtue of their exit pinhole. By closing the pinhole down, you can capture just the tip of the airy disk, and achieve a resolution greater than that available from a wide-field microscope. This reduces the amount of light collected, and therefore results in noisier images. However, you can scan the image more slowly to increase the exposure time for each pixel, resulting in a less noisy image. The downside of this is that you might kill or bleach your sample in just a few scans because of the intensity of the illumination. Again, it is a trade-off.

Deconvolution systems can circumvent this problem. Either in conjunction with a confocal or by using a wide-field microscope, the deconvolution system can yield an increased resolution.

The benefit of using deconvolution in conjunction with a confocal (any type of confocal) is that it allows you to use a larger exit pinhole, and hence collect more light. This allows lower illumination intensities and results in reduced photo-damage while achieving a high image resolution.

Deconvolution can also significantly improve images from a wide-field microscope, provided the sample is relatively sparse. Deconvolution systems tend to be less effective on samples containing large amount of diffuse fluorescence.

\section{Photo-Damage}

As noted above, deconvolution techniques can reduce photodamage by allowing you to decrease illumination intensity.

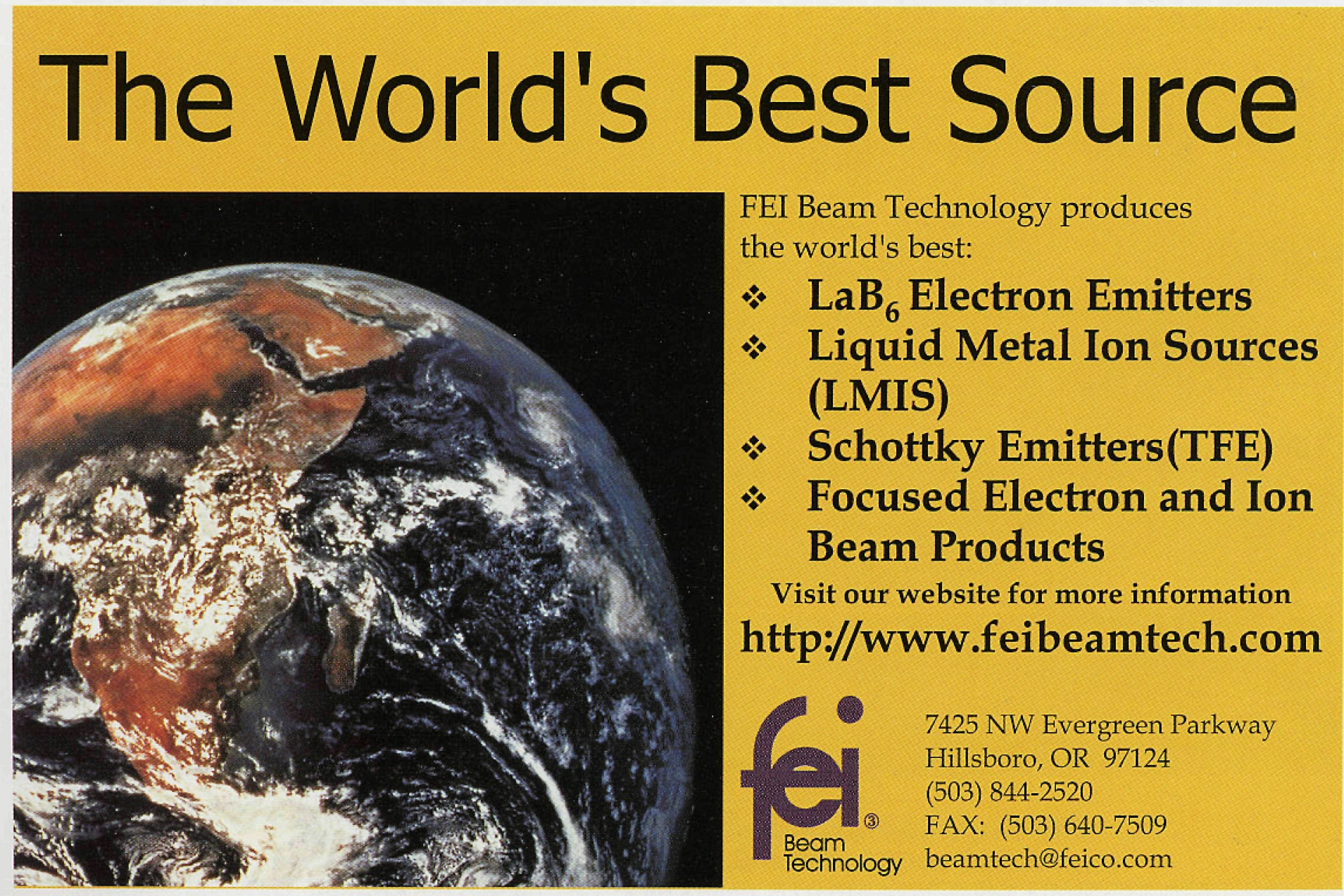


While there is still some debate on this topic, some claim that two photon confocals result in reduced photo-damage when compared to their single photon brethren. The reduction arises from the fact that two photon systems excite the sample using long wavelength light. Because longer wavelength photons have less energy, they typically damage the samples less.

However, this too must be cautiously stated. If parts of the sample absorb long wavelength light, the damage can be dramatic. The word "vaporization" comes to mind.

\section{Excitation Wavelengths}

In wide-field fluorescence microscopy, we take it for granted that it is a simple matter to use any desired fluorochrome. Unfortunately, with confocal microscopes, the supplied laser that is used to excite the fluorechrome limits the choice of excitation wavelengths.

In this respect, deconvolution systems have a huge advantage over laser based confocals, because anything that can be visualized can be deconvolved, deconvolution is virtually unlimited in its use of excitation wavelengths.

\section{Ease of Use}

Quite often, highly technical people choose confocal microscope systems to be used by a larger group. They may evaluate the system thoroughly, based on a variety of technical criteria such as those listed above. However, if others in the group will be using the instrument, then it is absolutely necessary to evaluate it based on its ease of use.

An interesting, but believable, rumor is that the majority of the conventional, point scanning confocal microscopes sold in Japan are unused because there are not enough users who can actually operate them! What do they use instead?

The spinning disk confocal, with its real-time direct viewing and its simplicity of operation stands apart from all other currently available confocal technologies. The simple reason for its ease of use is that a computer isn't necessary. You simply turn on the laser and the motor that runs the spinning disk. No configuration. No alignment. You simply look through an eyepiece to see the confocal image.

The spinning disk confocal simplifies use in another significant way. Because it produces a real-time image, you can scan and focus to find your sample just as you would a normal microscope. Anyone who has tried this with a conventional confocal understands how liberating this feature can be.

Some other confocal designs do permit real-time image viewing. The resonant galvo point scanner and the line scanner both benefit from this advantage.

\section{Conclusions}

This article covered a variety of factors to consider when choosing a confocal microscope. These included speed, resolution, photo-damage, excitation wavelengths and ease of use. In addition, it included discussion of how computational deconvolution can improve images from any scope confocal or wide-field, showing that these are far more complementary technologies than has been previously argued.

While by no means exhaustive, these few items can help you prioritize your usage needs and enable you to choose the best imaging technologies for your applications.

Handbook of Biological Confocal Microscopy, J.B. Pawley, editor, 2nd edition, Plenum Press, New York, 1995

3-D Laser Sanning Confocal Microscopy Page - http://www.cs.ubc.ca/ spider/ladic/confocal.html

Three-Dimensional Deconvolution of Optical Microscope Images Using Adaptive Inverse Modeling - http://panda.uchc.edu/htbit/home_pages/ inverse_filter.html

Advantage of Two-Photon Excitation Imaging - http://www.mc.vanderbilt. edu/vumcdept/mpb/piston/files/2padvan.html

Integrated Microscopy of Gene Transfer and Expression - http://www. bocklabs.wise edu/imr/integrat/transg. htm

Confocal Microscopy -

http://www.med.ohio-state.edu/cmif/confocal.htm

Prospects for Multi-photon Microscopy - http://journals.wiley.com/ cytometry/ISAC98/2MAEAmos.htm

Introduction to Optics and Confocal Microscopy - http://lucifier.life.utsa. edu/CLSM/chpt2.html

Best Quality, Best Price Microscope Bulbs

Osram Mercury HBO 100, only $\$ 107.99$

Osram Mercury HBO 103, only $\$ 116.99$

Osram Xenon XBO75 Ozone Free, only $\$ 132.99$

2 bulb minimum - add $\$ 5.00$ per bulb for single orders

Shipping/handling/insurance from stock only $\$ 4.95$

Telephone or On-Site Technical Support Upon Request: $\$ 42.50$

Best Quality, Best Price Objective Repair

Inside/Outside Oil Removal per Objective only $\$ 59.99$

If not repairable, shop fee only $\$ 24.99$

Return shipping/handling/insurance $\$ 14.95$

\section{Sciscope Instrument Company} Leica Kodak Sony Osram Dealer

Mail orders: Box 2237, lowa City, lowa 52244 USA USA telephone orders: 1-800-55-MICRO

Overseas telephone orders: +319-338-1107

Fax orders: 1-319-338-3954 Internet orders: www.sciscope.com

* Include your Visa, Master Card or American Express information

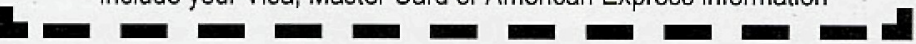

\section{WinEDS 3.0 High Performance X-ray Microanalysis For Windows ${ }^{\mathrm{TM}}$ 95 / NT 4.0}

This FAST full 32-bit PC Windows ${ }^{T M}$ based analyzer gives you all the performance of more expensive workstation systems.

* Qualitative \& Quantitative software with one click operation.

* WinEDS PX9000 Pulse Processor DX3000 Bias.

X Interfaces to several detector brands.

X 5 to $10 \mathrm{eV}$ improvement on most detector's resolutions.

X Complete system upgrades - including new detectors.

X Partial upgrades to suit your system needs. Also

* Complete line of PC based imaging systems.

* Top dollar for trade-ins.

X Service for TN5500 and TN2000 systems.

\section{TN Analyzer Service Inc.} 7897 Hwy 19

Dane, WI 53529

Tel.: (608)798-2005, Fax: (608)798-1675, eMail: doug_tnas @msn.com

Visit our web site: www.tnanalyzerservice.com

Now with demo labs in Florida and Texas, as well as in Wisconsin - and soon on the west coast. 